\title{
The B-dot Earth Average Magnetic Field
}

\author{
By \\ Pedro A. Capo-Lugo, John Rakoczy \\ EV41, Control System Design \& Analysis Branch \\ Devon Sanders \\ EV42, Guidance, Navigation and Mission Analysis Branch \\ NASA Marshall Space Flight Center \\ Huntsville, Alabama 35812
}

The average Earth's magnetic field is solved with complex mathematical models based on mean square integral. Depending on the selection of the Earth magnetic model, the average Earth's magnetic field can have different solutions. This paper presents a simple technique that takes advantage of the damping effects of the b-dot controller and is not dependent of the Earth magnetic model; but it is dependent on the magnetic torquers of the satellite which is not taken into consideration in the known mathematical models. Also the solution of this new technique can be implemented so easily that the flight software can be updated during flight, and the control system can have current gains for the magnetic torquers. Finally, this technique is verified and validated using flight data from a satellite that it has been in orbit for three years.

\section{Nomenclature}

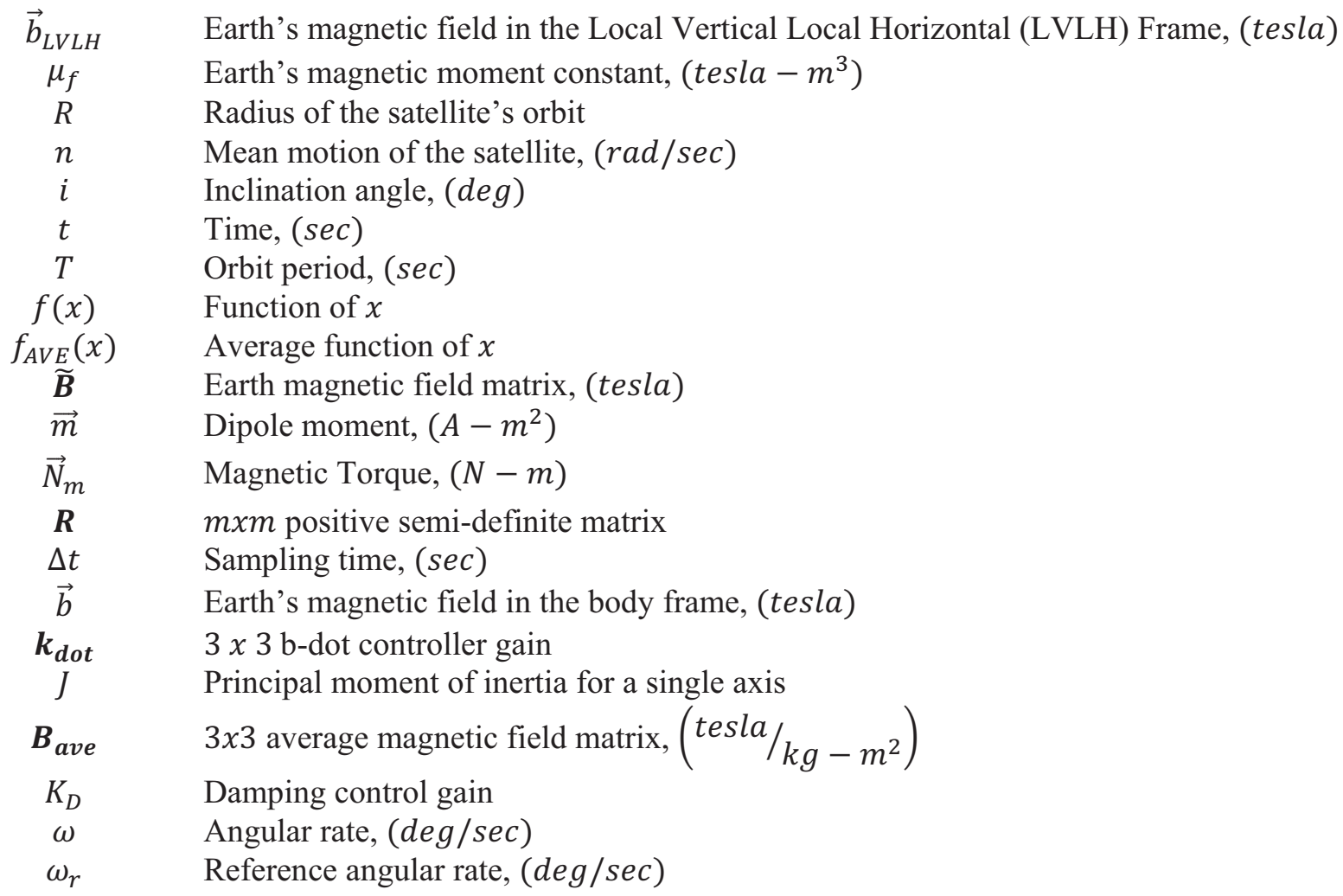




$\begin{array}{cl}\xi & \text { Damping coefficient } \\ \tau & \text { Number of orbits or the ratio between the time and the orbital period } \\ s & \text { Laplace transform variable } \\ y & \text { Function for the correlation data } \\ y_{0} & \text { Initial condition for the correlated data } \\ m & \text { Decaying coefficient for the correlated data } \\ J_{x x}, J_{y y}, J_{z z} & \text { Principal moments of inertia for roll, pitch, and yaw, respectively, }\left(\mathrm{kg}-\mathrm{m}^{2}\right) \\ \boldsymbol{J} & \text { Principal moments of inertia matrix, }\left(\mathrm{kg}-\mathrm{m}^{2}\right) \\ \boldsymbol{I} & \text { Identity matrix }\end{array}$

\section{Introduction}

The average magnetic field is commonly used in control schemes [1] to simplify the control gain calculation because the Earth's magnetic field is a periodic function. Lovera [2] showed that the average Earth's magnetic field for the dipole model is defined as a closed-form solution. On the other hand, Psiaki [1] uses a very similar calculation to average the Earth's magnetic field in his control scheme. Also, the average Earth's magnetic field can be determined by numerical integration which shows a different solution in compare to the closed-form solution.

The purpose of this paper is to present a different method to determine the average Earth's magnetic field. This method is easily implemented by using the International Geomagnetic Reference Field (IGRF) and the b-dot controller. By combining these two models, it is shown that the average value of the Earth's magnetic field is constant in any of the three axes of the satellite. Also, this method can be implemented with the flight data so that an update to the flight software can be transmitted to the satellite during flight. To validate this method, the Fast and Affordable Science and Technology Satellite (FASTSAT) flight data is used.

This paper begins by explaining the available Earth's magnetic field models and the solution for the different calculation of the average Earth's magnetic field. Then, it continues explaining the new method using the b-dot controller. In addition, the FASTSAT mission is explained and used to simulate the new method to determine the average magnetic field. Finally, a validation procedure is performed to check the solutions obtained with the new method.

In conclusion, this paper will demonstrate the effectiveness to determine the average value of the Earth's magnetic field with the b-dot controller. Also, it will demonstrate how the average value can be determined from the flight data so that the flight software can be updated in-orbit.

\section{Earth's Magnetic Field Model}

There are different models to describe the Earth's magnetic field. The Earth's magnetic field can be described as a dipole model, a tilted-dipole model [3], and the International Geomagnetic Reference Field Model (IGRF) [4]. For a spacecraft in a circular orbit, the dipole model is commonly used to determine the control gains. For the dipole model, the Earth's magnetic model [5] is described as, 


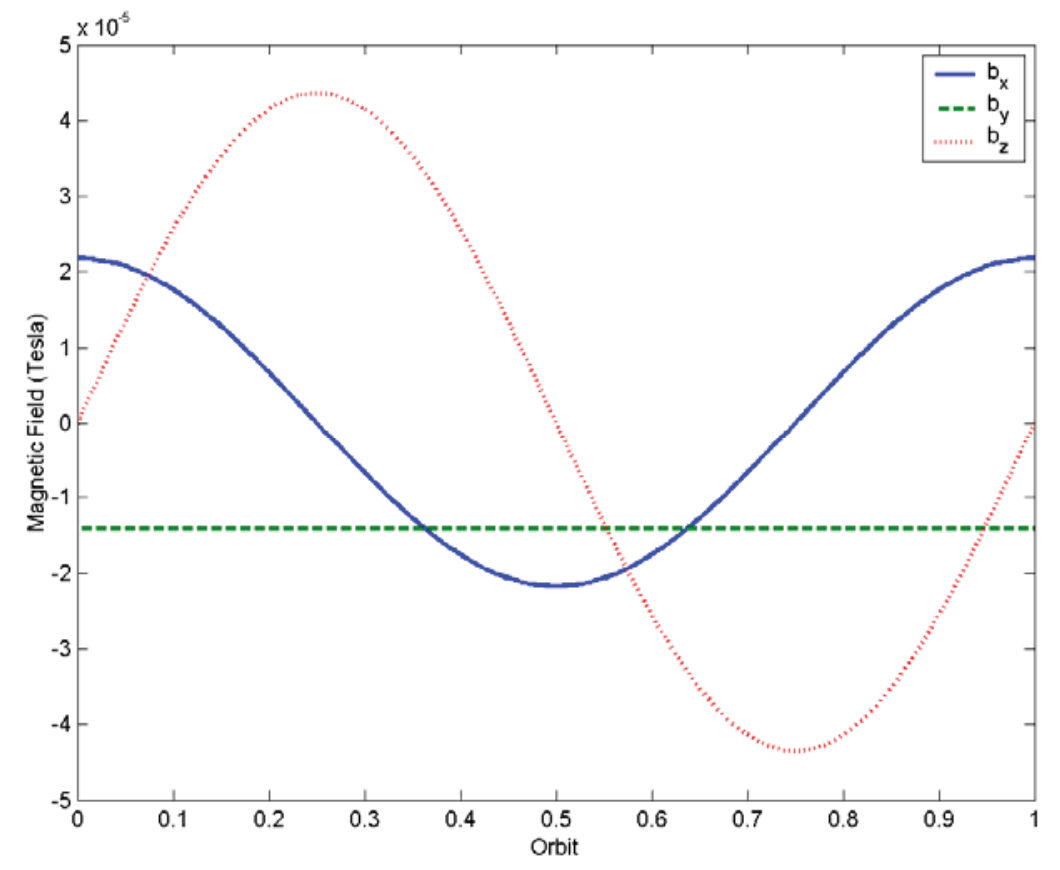

Figure 1. Earth's Magnetic Dipole

$$
\vec{b}_{L V L H}=\frac{\mu_{f}}{R^{3}}\left[\begin{array}{c}
\cos n t \sin i \\
-\cos i \\
2 \sin n t \sin i
\end{array}\right]
$$

$\mu_{f}$ is the Earth's magnetic moment equal to $7.95 e^{15}\left(\right.$ tesla $\left.-m^{3}\right), R$ is the radius of the circular orbit measured from the center of the Earth, and $i$ is the inclination of the orbit. $n$ is the mean motion of the satellite described as,

$$
n=\frac{2 \pi}{T}
$$

where $T$ is the period of the orbit. Figure 1 shows the dipole model of the Earth's magnetic field for one orbit period assuming a satellite at an altitude of $650 \mathrm{~km}$ and an inclination angle of $72^{\circ}$. In Figure 1, the $y$ axis of the Earth's magnetic field is always constant while the $x$ and $z$ axis changes with respect to the satellite location.

To simulate the controller, it is preferred to use an updated model of the Earth's magnetic field such as the IGRF models. Figure 2 shows the output of the IGRF-10 model [4] [6] for the same satellite as in Figure 1. There are noticeable differences between Figure 1 and Figure 2 such as the $y$ axis is not constant in the IGRF-10 model, and the IGRF-10 does not behave as a sinusoidal function. When the IGRF-10 is propagated for more than one orbit, the IGRF models behave as a periodic function. 


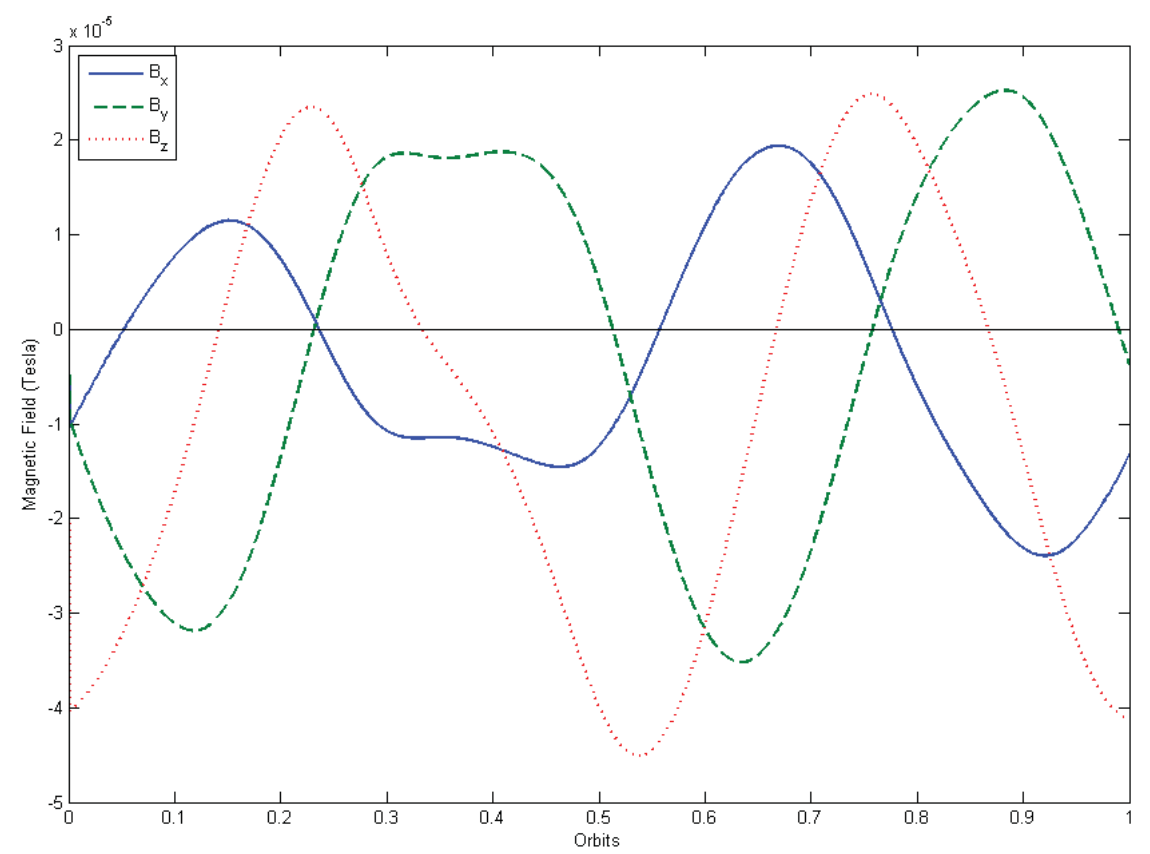

Figure 2. Earth's Magnetic Dipole

\section{Average Magnetic Field in the Literature}

Many active control schemes [1] [7] [8] use the average and the periodic effect of the magnetic field to determine the gains and the stability of the controller. This section explains a few methods utilized in the literature to determine the average magnetic field.

The average value of a function can be determined as,

$$
f_{A v g}=\frac{1}{b-a} \int_{a}^{b} f(x) d x
$$

Because the Earth's magnetic field is periodic, the following property can be used, $f(t+T)=f(t)$ where $T$ is the period of the function. Using this property, the average value for the periodic function is,

$$
f_{\text {Avg }}=\frac{1}{T} \int_{0}^{T} f(t) d t
$$

As shown in Equation (1), the Earth's Magnetic field is described in terms of sine and cosine functions which are periodic; if $f(t)$ is represented by a sine and/or cosine function, the average function in equation (3) is equal to zero.

$$
f_{A v g}=\frac{1}{T} \int_{0}^{T} f(\cos (n t), \sin (n t)) d t=0
$$

On the other hand, the average value is obtained by the mean square error of the function which is described as, 


$$
f_{\text {Avg }}=\frac{1}{T} \int_{0}^{T}|f(\cos (n t), \sin (n t))|^{2} d t
$$

It is shown through the literature [9] that the root mean squared value of a sine or cosine function is 0.707 of the amplitude of the function. Even though the solution for the mean square error is known, the average magnetic field is determined from the magnetic torque equation written in state-based format as,

$$
\boldsymbol{J}^{-1} \vec{N}_{m}=-\boldsymbol{J}^{-1} \vec{b}_{E C I} \times \vec{m}=\widetilde{\boldsymbol{B}} \vec{m}
$$

where,

$$
\widetilde{\boldsymbol{B}}=\left[\begin{array}{ccc}
0 & \frac{b_{3}}{J_{x x}} & -\frac{b_{2}}{J_{x x}} \\
-\frac{b_{3}}{J_{y y}} & 0 & \frac{b_{1}}{J_{y y}} \\
\frac{b_{2}}{J_{z z}} & -\frac{b_{1}}{J_{z z}} & 0
\end{array}\right] \quad \vec{m}=\left[\begin{array}{l}
m_{1} \\
m_{2} \\
m_{3}
\end{array}\right]
$$

$\boldsymbol{J}$ is the principal moments of inertia of the satellite. The average magnetic field was determined in Reference [2] and [10] with equations (4) and (5) assuming that the Earth's Magnetic field is described by the magnetic dipole model. Lovera [2] said that the closed-form solution of the average magnetic field for the Earth's magnetic dipole is,

$$
\frac{1}{T} \int_{0}^{T} \widetilde{\boldsymbol{B}} \widetilde{\boldsymbol{B}}^{T} d t=\left(\frac{\mu_{f}}{R^{3}}\right)^{2}\left[\begin{array}{ccc}
\frac{\cos ^{2} i+2 \sin ^{2} i}{J_{x x}^{2}} & 0 & 0 \\
0 & \frac{\frac{5}{2}\left(\sin ^{2} i\right)}{J_{y y}^{2}} & 0 \\
0 & 0 & \frac{\cos ^{2} i+\frac{1}{2} \sin ^{2} i}{J_{z z}^{2}}
\end{array}\right]
$$

For Lovera [2], the average magnetic field is not dependent on the satellite orbit position but is dependent on the inclination angle. In the controller, the orbit position has a big role in the controller because the magnetic torquers could provide an additional torque in another axis (see equation (5)). Also, it can be observed that the average value is a maximum when the satellite is in a polar orbit.

On the other hand, Psiaki [1] estimates the average magnetic field from the linear quadratic regulator (LQR) control law. In the LQR, the Riccati equation contains a $\widetilde{\boldsymbol{B}} \widetilde{\boldsymbol{B}}^{T}$ multiplication which makes the Riccati matrix periodic. Even though the average for Lovera [2] and Psiaki [1] are very similar, the Riccati equation shows a multiplication by a constant matrix within $\widetilde{\boldsymbol{B}} \widetilde{\boldsymbol{B}}^{T}$. Psiaki [1] represents the average magnetic field as,

$$
\frac{1}{T} \int_{0}^{T} \widetilde{\boldsymbol{B}} \boldsymbol{R}^{-\mathbf{1}} \widetilde{\boldsymbol{B}}^{T} d t
$$


$\boldsymbol{R}$ is a positive semi-definite $m \times m$ matrix describing the weight of the control input and is commonly written as a principal diagonal matrix.

However, the integration can be performed numerically in which the total integral is the accumulative sum over one period. If this integration is performed, equation (7) can be approximated to,

$$
\frac{1}{T} \int_{0}^{T} \widetilde{\boldsymbol{B}} \boldsymbol{R}^{-\mathbf{1}} \widetilde{\boldsymbol{B}}^{T} d t=\frac{\Delta t}{T} \sum \widetilde{\boldsymbol{B}} \boldsymbol{R}^{-\mathbf{1}} \widetilde{\boldsymbol{B}}^{T}
$$

where $\Delta t$ is the sampling time of the integration procedure. The numerical integration procedure in equation (8) will produce a different result as shown in equation (4). The integration of equation (4) as a continuous function disregards the effects of the orbit position because it is a periodic function. In equation (8), the average value is accumulated through the entire period in which the off-diagonal terms of the average magnetic field are not zero. Although equation (4) provides a closed-form solution for the dipole model, equation (8) can be used for the tilted dipole model and the IGRF models to determine the average value.

\section{B-Dot Average Magnetic Field}

Taking the time derivative of the magnetometer measurement in the body frame, the magnetic field rate is related to the attitude rate of the satellite [3]. If the time derivative of the magnetic field is multiplied by a constant gain, the dipole moment of the magnetic controller can be determined as,

$$
\vec{m}=-\boldsymbol{k}_{\boldsymbol{d o t}} \dot{\vec{b}}
$$

where,

$$
\boldsymbol{k}_{\text {dot }}=\left[\begin{array}{ccc}
k_{d o t, x} & 0 & 0 \\
0 & k_{d o t, y} & 0 \\
0 & 0 & k_{d o t, z}
\end{array}\right]
$$

Equation (9) is known as the b-dot controller, and $\boldsymbol{k}_{\boldsymbol{d o t}}$ is the gain matrix. The controller is continuously used through the entire orbit to damp the attitude rates to two revolutions per orbit, and this controller does not provide means to control the attitude motion because it only depends on the rates of the satellite. Figure (3a) shows a block diagram for the b-dot controller.

The purpose of the b-dot controller is to calculate a dipole moment to reduce the attitude rates in a varying magnetic field. Instead, an average value of the Earth Magnetic field times a constant gain can also be used to reduce the rates of the satellite. Figure (3b) shows a simplified block diagram of the b-dot controller in the average Earth's magnetic field. The b-dot controller is better described in terms of the number of orbits because of the time that the controller takes to damp the rates. Knowing that $\tau={ }^{t} / T$ and using the scaling theorem of the Laplace transform [11], the transfer function is written as,

$$
\frac{\omega(s T)}{\omega_{r}(s T)}=\frac{K_{D} B_{\text {ave }} / J T}{s+{ }^{K_{D} B_{\text {ave }} / J T}}=\frac{\xi / T}{s+\xi / T}
$$




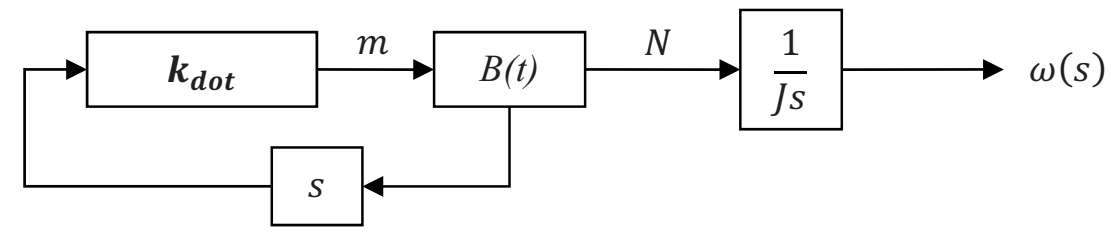

(a)

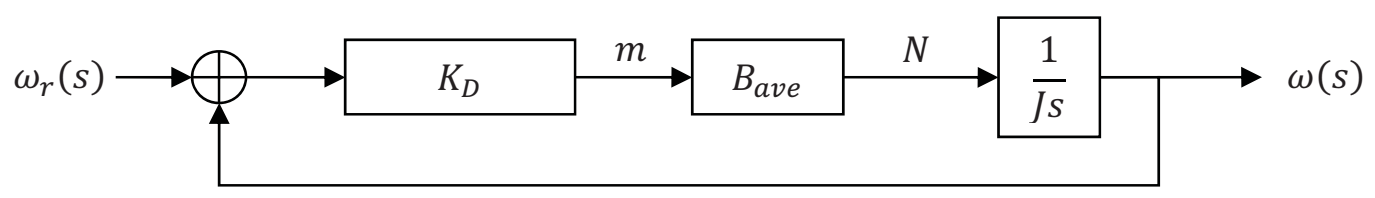

(b)

Figure 3. Block diagrams for b-dot controller for (a) varying magnetic field and (b) average magnetic field

where $\omega$ is the angular velocity of the spacecraft, $\omega_{r}$ is the reference angular velocity, $K_{D}$ is the derivative gain of the controller, $B_{\text {ave }}$ is the average value of the magnetic field, and $J$ is the principal moment of inertia about a single reference axis in the satellite. $\tau$ describes the number of orbits that the satellite takes to decay the attitude rates. The derivative gain of the b-dot controller has to be divided by the term $\mu_{f} / R^{3}$ because the b-dot controller will not damp the rates for values lower than $\mu_{f} / R^{3}$. The derivative gain of the $b$-dot controller can be written as,

$$
\boldsymbol{k}_{\boldsymbol{d o t}}=\frac{R^{3}}{\mu_{f}} K_{D} \boldsymbol{I}
$$

where $\boldsymbol{k}_{\boldsymbol{d o t}}$ is the gain applied to the b-dot controller, and $\boldsymbol{I}$ is a $3 \times 3$ identity matrix. For now, it will be assumed that $\boldsymbol{k}_{\boldsymbol{d o t}}$ is the same along the principal diagonal.

Solving the Laplace equation (10), the angular rate in the time domain is,

$$
\omega(\tau)=\frac{\xi}{T} e^{-\xi \tau} \omega_{r}
$$

and $\xi=K_{D} B_{\text {ave }} / \mathrm{J}$ which is the damping constant [12]; $\omega_{r}$ is the constant for the unit step function. The proof of this equation is shown in Appendix A. The damping value can be obtained by analyzing the bdot controller output for high attitude rates. A regression analysis [13] is performed to the body attitude rate data to correlate the points to a decaying exponential function. In order to do this, the b-dot controller will require high gains to damp the attitude rates in several orbits.

For an exponential regression line [13], the equation describing the estimated line can be written as,

$$
y(\tau)=y_{0} e^{-a \tau}
$$

where $y_{0}$ is the initial constant, and $a$ is the exponential decaying coefficient. The damping coefficient should be described in terms of orbits because the damping coefficient affects the angular rates of the 
satellite in the long term. When the linear regression is determined, the exponential decaying coefficient is calculated. Knowing this, equation (12) and equation (13) are compared to relate the coefficients as follows,

$$
y_{0}=\frac{\xi \omega_{r}}{T} \quad a=\xi=K_{D} B_{a v e} / J
$$

Since $a$ and $y_{0}$ is known, the following ratios are obtained,

$$
\begin{aligned}
& B_{\text {ave }} / J=\frac{a}{K_{D}} \\
& \omega_{r}=y_{0} T / \xi
\end{aligned}
$$

The important ratio is the one shown in equation (14a) because it describes the average magnetic field and is always used in different control schemes [1] [10] [14] [15]. Although $B_{\text {ave }}$ is shown to be different in any of the three axes of the satellite for the methods shown in the literature, this paper will demonstrate that the ratio $B_{\text {ave }} / J$ is constant in the three reference axis of the satellite using this new method. In addition, the constant $B_{\text {ave }} / J$ can be updated in the controller so that the flight software can be updated with a new set of gains.

\section{Study Case}

The Fast and Affordable Science and Technology Satellite (FASTSAT) [16] was launched in 2010 into a $650 \mathrm{~km}$ orbit at an inclination angle of $72^{\circ}$. The spacecraft carried several science instruments developed by Goddard Space Flight Center (GSFC), U.S. Naval Academy, and Marshall Space Flight Center (MSFC) [16]. FASTSAT had a mass of $150 \mathrm{~kg}$, and the principal moments of inertia were approximated to,

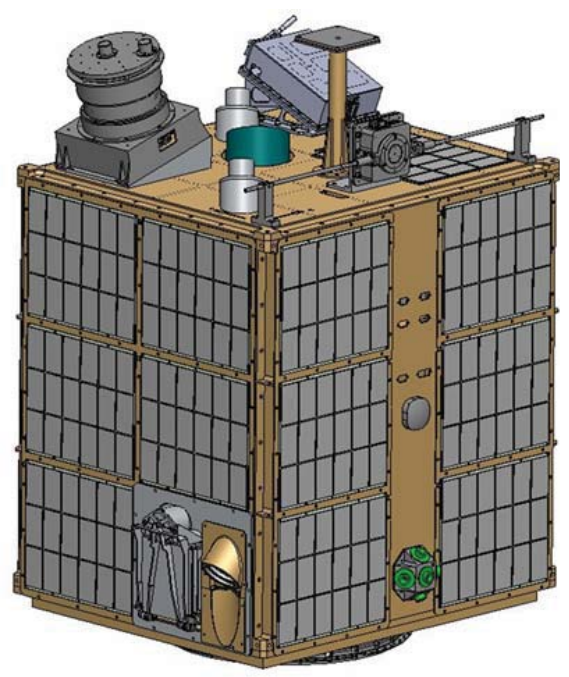

Figure 4. Fast Affordable Science and Technology Satellite (FASTSAT) 


$$
\left[\begin{array}{l}
J_{x x} \\
J_{y y} \\
J_{z z}
\end{array}\right]=\left[\begin{array}{l}
15.9 \\
16.9 \\
11.7
\end{array}\right]\left(k g-m^{2}\right)
$$

Figure 4 illustrates a computational aid designed (CAD) drawing after integration. This satellite had three magnetic torque rods oriented orthogonally and placed at one corner of the satellite. Each torquer rod was capable of producing up to $5\left(A-m^{2}\right)$ of dipole moment. Within the satellite's attitude determination hardware, it had a 3 axis magnetometer used to measure the magnetic field in the body frame. These mass properties and attitude control hardware will be used to study the average Earth magnetic field.

\section{Literature Average Magnetic Field}

This section determines the average magnetic field for the different cases found in the literature using the Earth magnetic dipole model and the IGRF. The FASTSAT orbit is used to determine the average value of the Earth magnetic field for the different cases. The following results show the mean-squared error of the Earth's magnetic field found in the literature:

1. Lovera [2] Earth Magnetic Closed-Form Solution (Dipole Model)

$$
\begin{gathered}
\boldsymbol{B}_{\text {ave }} \boldsymbol{B}_{\text {ave }}^{T}= \\
=\frac{1}{T} \int_{0}^{T} \widetilde{\boldsymbol{B}} \widetilde{\boldsymbol{B}}^{T} d t=\left[\begin{array}{ccc}
4.472 \times 10^{-12} & 0 & 0 \\
0 & 4.618 \times 10^{-12} & 0 \\
0 & 0 & 2.214 \times 10^{-12}
\end{array}\right]\left(\frac{\text { teslas }}{\mathrm{kg}-\mathrm{m}^{2}}\right)^{2} \\
\boldsymbol{B}_{\text {ave }}=\left[\begin{array}{ccc}
2.115 \times 10^{-6} & 0 & 0 \\
0 & 2.149 \times 10^{-6} & 0 \\
0 & 0 & 1.488 \times 10^{-6}
\end{array}\right]\left(\frac{\text { teslas }}{\mathrm{kg}-\mathrm{m}^{2}}\right)
\end{gathered}
$$

2. Integral Model (Dipole Model and $\boldsymbol{R}=\boldsymbol{I}$ )

$$
\begin{gathered}
\boldsymbol{B}_{\text {ave }} \boldsymbol{B}_{\text {ave }}^{T}=\frac{1}{T} \int_{0}^{T} \widetilde{\boldsymbol{B}} \widetilde{\boldsymbol{B}}^{T} d t=\left[\begin{array}{ccc}
4.416 \times 10^{-12} & 3.955 \times 10^{-29} & 1.565 \times 10^{-28} \\
3.955 \times 10^{-29} & 4.561 \times 10^{-12} & -9.536 \times 10^{-29} \\
1.565 \times 10^{-28} & -9.536 \times 10^{-29} & 2.187 \times 10^{-12}
\end{array}\right]\left(\frac{\text { teslas }}{\mathrm{kg}-\mathrm{m}^{2}}\right)^{2} \\
\boldsymbol{B}_{\text {ave }}=\left[\begin{array}{ccc}
2.101 \times 10^{-6} & 9.670 \times 10^{-21} & 1.353 \times 10^{-22} \\
9.670 \times 10^{-21} & 3.5281 \times 10^{-6} & 3.467 \times 10^{-7} \\
1.353 \times 10^{-22} & 3.467 \times 10^{-7} & 8.633 \times 10^{-6}
\end{array}\right]\left(\frac{\text { teslas }}{\mathrm{kg}-\mathrm{m}^{2}}\right)
\end{gathered}
$$

3. Integral Model(IGRF-10 Model and $\boldsymbol{R}=\boldsymbol{I})$

$$
\begin{aligned}
\boldsymbol{B}_{\text {ave }} \boldsymbol{B}_{\text {ave }}^{T}= & \frac{1}{T} \int_{0}^{T} \widetilde{\boldsymbol{B}} \widetilde{\boldsymbol{B}}^{T} d t \\
& =\left[\begin{array}{ccc}
1.586 \times 10^{-10} & 3.0 \times 10^{-11} & -3.021 \times 10^{-11} \\
3.0 \times 10^{-11} & 1.046 \times 10^{-10} & -2.152 \times 10^{-11} \\
-3.021 \times 10^{-11} & -2.152 \times 10^{-11} & 1.808 \times 10^{-10}
\end{array}\right]\left(\frac{\text { teslas }}{\mathrm{kg}-\mathrm{m}^{2}}\right)^{2} \\
\boldsymbol{B}_{\text {ave }} & =\left[\begin{array}{ccc}
1.248 \times 10^{-5} & 1.286 \times 10^{-5} & -1.126 \times 10^{-6} \\
1.286 \times 10^{-6} & 1.11 \times 10^{-5} & -8.548 \times 10^{-7} \\
-1.126 \times 10^{-6} & -8.548 \times 10^{-7} & 1.337 \times 10^{-5}
\end{array}\right]\left(\frac{\text { teslas }}{\mathrm{kg}-\mathrm{m}^{2}}\right)
\end{aligned}
$$


I is the identity matrix. The dipole model always provides a principal diagonal matrix because of the assumptions made in the model. On the other hand, the integral model provides a fully populated matrix in which the off-diagonal are the same as the principal diagonal of the dipole model if approximated. Using the IGRF model, the matrix is also fully populated, but the principal diagonal of the integral model with the IGRF-10 is relatively larger than the principal diagonal in the integral model with the dipole model. In summary, the closed-form solution provides an upper bound to the solution of the average Earth's magnetic field, but the average magnetic field with the IGRF model should be used because the off-diagonal terms creates a coupling in the solution not observed in the dipole model.

\section{B-dot Average Magnetic Field - Case Study}

The initial attitude for the satellite simulation can be arbitrary, but it is recommended to use high attitude rates to analyze the damping effects in the b-dot controller. For this case study, the attitude motion is setup so that the initial attitude angles are zero and the initial attitude rates are $3 \mathrm{deg} / \mathrm{sec}$ in the pitch axis and $1 \mathrm{deg} / \mathrm{sec}$ in the roll and yaw axes. In addition, $K_{D}=200,000$ for the three axes of the satellite. Figure 5 shows the simulated b-dot control solution for FASTSAT for this set of initial conditions. The top, middle, and bottom graphs are, respectively, the roll, pitch, and yaw attitude rates in the satellite's body frame. As shown in Figure 5, the attitude rates are damped in 2.5 orbits.
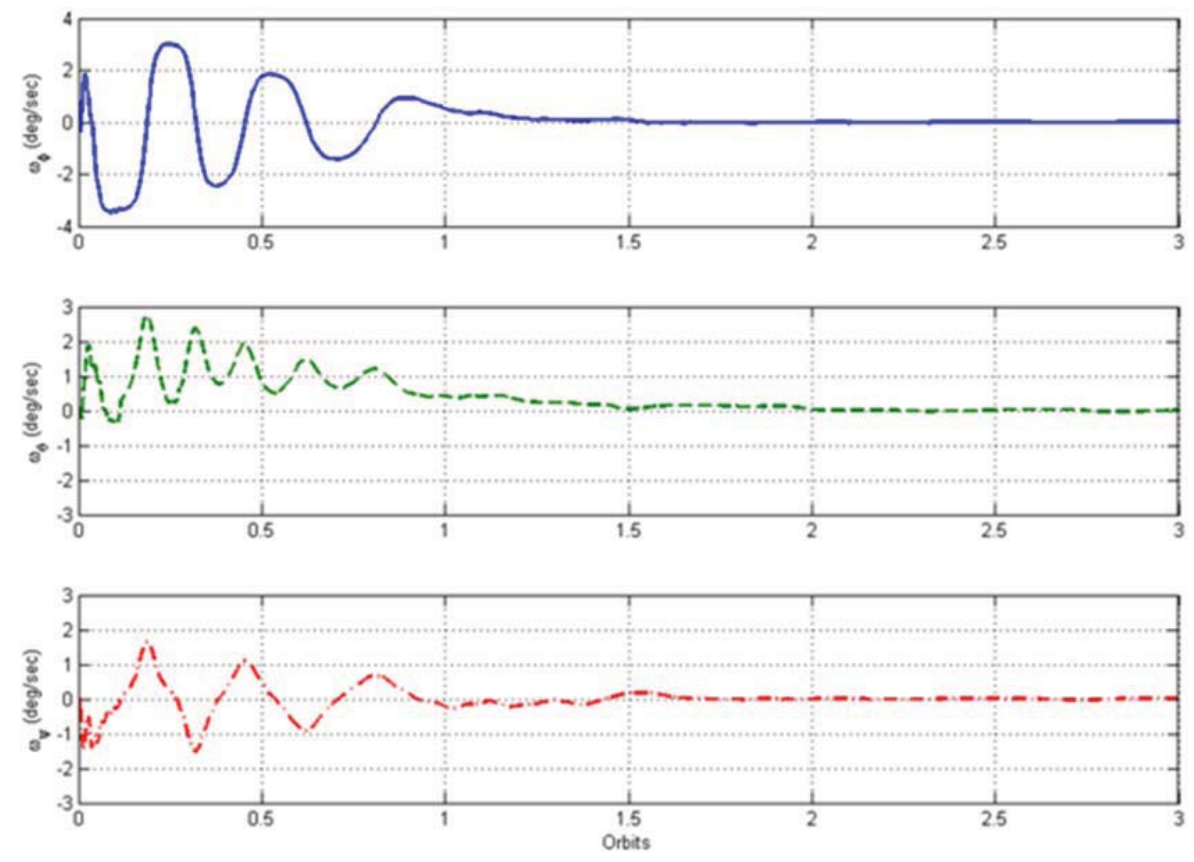

Figure 5. Angular velocity of a satellite using a b-dot controller 


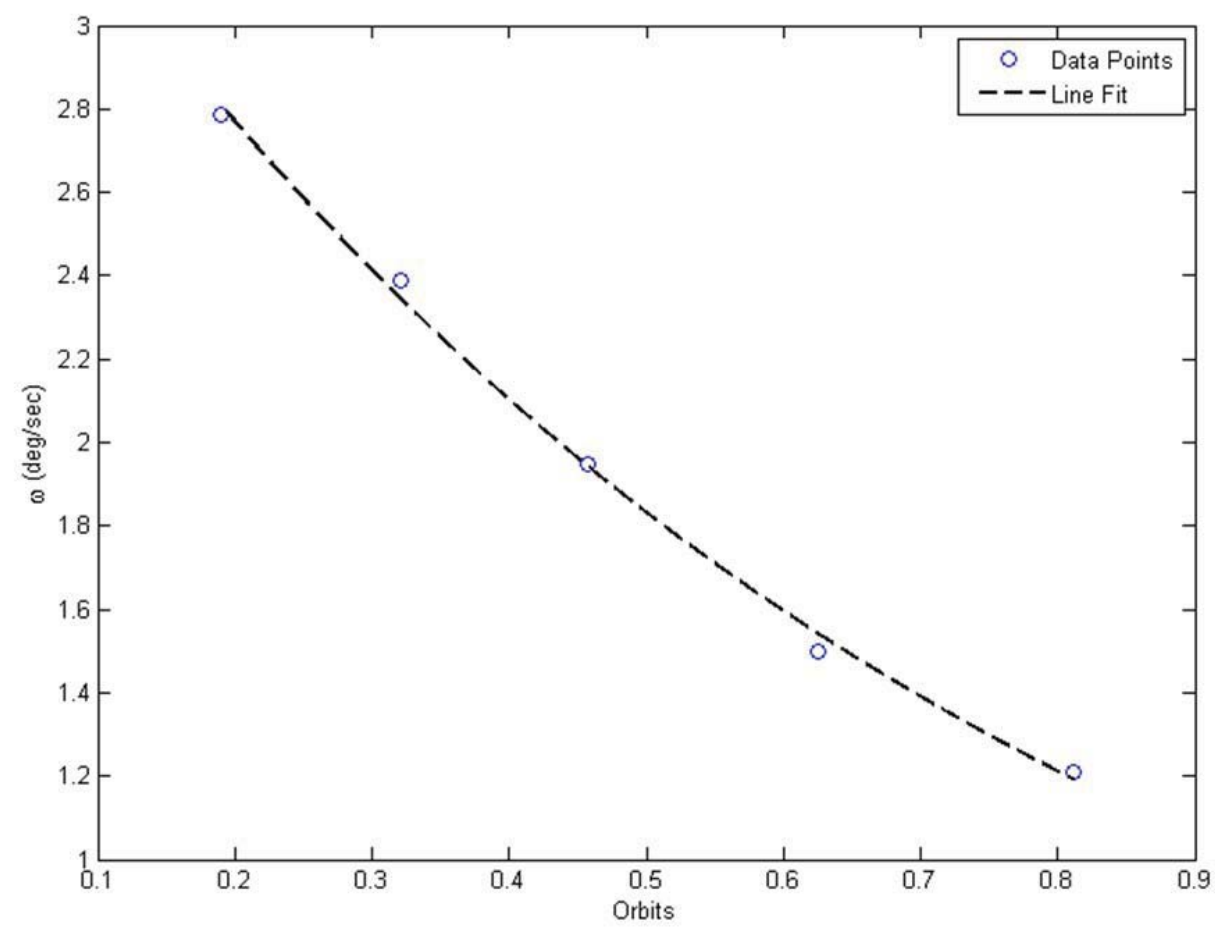

Figure 6. Regression analysis for the damping coefficient (simulation)

The average magnetic field, using the b-dot controller, can be obtained from any of the three curves shown in Figure 5. Using the pitch axis (middle graph) in Figure 5, the positive maximum point at the crest of every wave is selected. This value is plotted in Figure 6 to determine the exponential decaying function. The exponential function is determined by regression analysis. The ratio in equation (14a) describing the average magnetic field for Figure 5 are equal to,

$$
B_{\text {ave }} /_{I}=a / K_{D}=6.897 \times 10^{-6}\left(\frac{\text { teslas }}{\mathrm{kg}-\mathrm{m}^{2}}\right)
$$

If the same analysis is performed for the other two axes, the results are the same as in equation (15). Hence, equation (15) provides the average of the magnetic field per moment of inertia of the satellite. The results in equation (15) show a very similar solution as in Section VI, but the $B_{\text {ave }}$ is a value larger than the solutions shown in the literature (Lovera [2] and Psiaki [1]). The difference between this new method and the mean square of the magnetic field is that this new method takes into consideration the rate damping effects of the magnetic torquers.

The following steps can be generalized to determine the average magnetic field:

1. Select a value for $K_{D}$ to determine $\boldsymbol{k}_{\boldsymbol{d o t}}$.

2. Use a high initial attitude rate for the three axes of the satellite ( $5 \mathrm{deg} / \mathrm{sec}$ is a good approximation).

3. Propagate the satellite orbit using the IGRF model and the b-dot controller. 

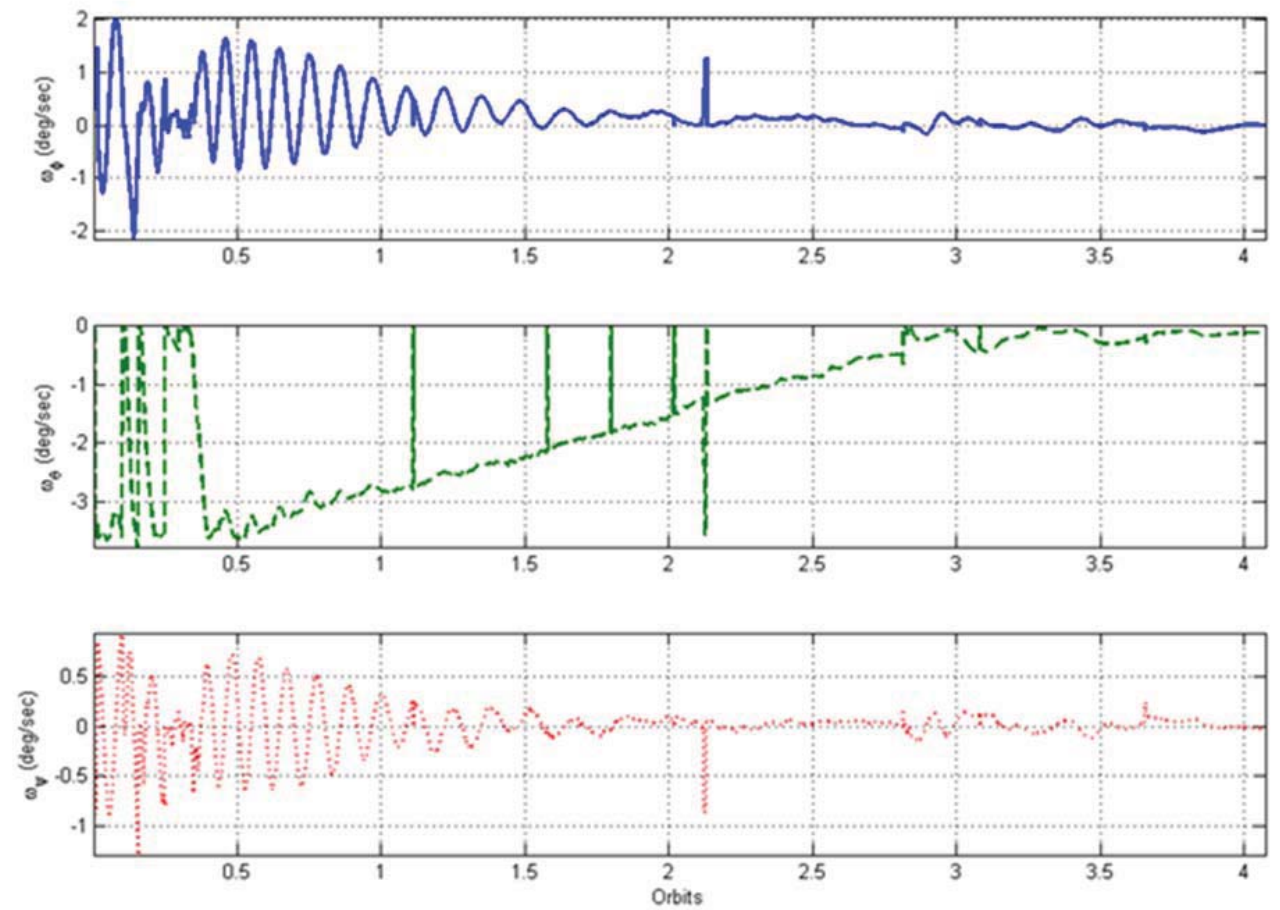

Figure 7. Tip-off rates using b-dot control (Flight Data)

4. Extract the maximum or minimum points of the angle rate data to determine the damping coefficient using regression analysis.

5. Use equation (14a) to determine the average Earth's magnetic field per principal moment of inertia.

\section{B-dot Average Magnetic Field Validation}

As mentioned before, FASTSAT was launched in 2010 into a circular orbit at $650 \mathrm{~km}$ altitude. The b-dot controller used the previous $K_{D}$ gain as the $\boldsymbol{k}_{\boldsymbol{d o t}}$ gain for the b-dot controller. Figure 7 shows the tip-off rates from the flight data of the satellite after deployed from the rocket. In Figure 7, the maximum tip-off rates are approximately $3 \mathrm{deg} / \mathrm{sec}$ about the pitch axis and $1 \mathrm{deg} / \mathrm{sec}$ about the roll and yaw axes. In approximately 4 orbits, the attitude rates are damped with the b-dot controller.

On the other hand, a simulation is performed to verify the performance of the derivative gain when $K_{D} \boldsymbol{I}=\boldsymbol{k}_{\boldsymbol{d o t}}$. When this gain is used, Figure 8 shows the results for the simulation. When Figure 7 and Figure 8 are compared, the rates in Figure 8 are not damped until 10 orbits while the flight data says that the rates were damped in 4 orbits. This is a big contradiction between the simulation and the flight data because $\boldsymbol{k}_{\boldsymbol{d o t}}<\frac{R^{3}}{\mu_{E}} \boldsymbol{I}$ where $\frac{R^{3}}{\mu_{E}}$ is the ratio between the orbit altitude and Earth's dipole moment shown in equation (1). If $\boldsymbol{k}_{\boldsymbol{d o t}}$ is specified as in equation (11), Figure 5 shows the simulation for the body attitude rates, and Figure 5 does not show a direct comparison to the rate damping shown in Figure 7 for FASTSAT. Although the time to do the rate damping is closely related between the simulation (Figure 5) 

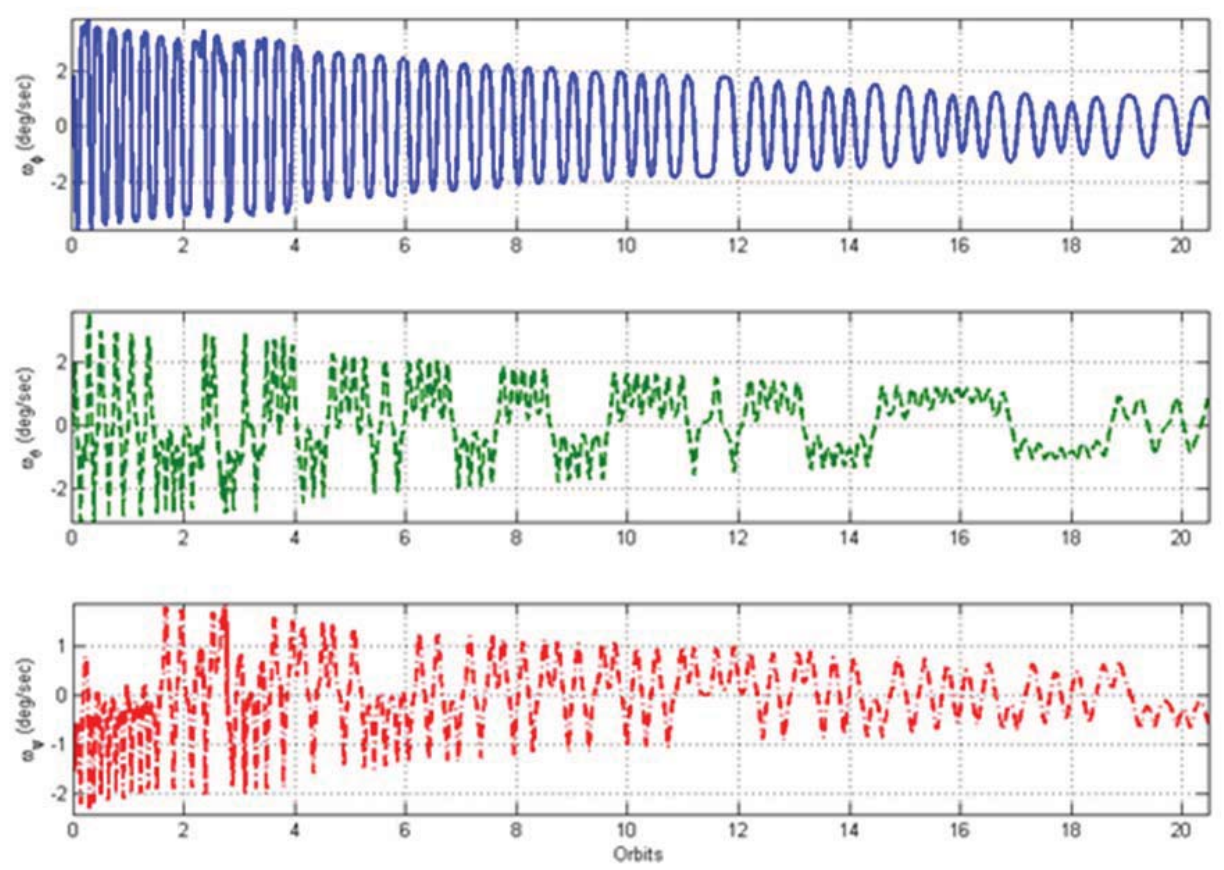

Figure 8. Simulated tip-off rates using b-dot control when $K_{D} \boldsymbol{I}=\boldsymbol{k}_{\boldsymbol{d o t}}$

and the flight data (Figure 7), the relation shown in equation (11) is validated to work in the simulation because $\boldsymbol{k}_{\boldsymbol{d o t}}>\frac{R^{3}}{\mu_{E}} \boldsymbol{I}$.

Finally, the average magnetic field per moment of inertia shown in equation (14) will be validated with the flight data. The three axes were analyzed to determine the average magnetic field. This paper will show the results for the roll axis because it shows a very nice damping coefficient near 0.5 orbits. Figure 9 shows the maximum values at each crest of the roll motion, and the dashed line is the regression line for the decaying exponential function. The damping coefficient, $a$, is equal to 1.412 . When $\xi$ is substituted into equation (14), the average magnetic field per moment of inertia equals to,

$$
B_{\text {ave }} /_{I}=7.060 \times 10^{-6}\left(\frac{\text { teslas }}{\mathrm{kg}-\mathrm{m}^{2}}\right)
$$

Comparing equation (15) with equation (16), there is an error of $2.4 \%$ in the solution. This means that the method shown here to develop the average magnetic field is validated against the flight data. In addition, the average magnetic field obtained through simulation provides a larger bound in comparison to the methods shown in the literature. The average magnetic field in equation (15) is determined using the pitch axis, but, in equation (16), the same analysis is performed with the flight data using the roll axis. The statement, that "the average magnetic field per moment of inertia is the same in three axes of the satellite is the same", is also validated. 


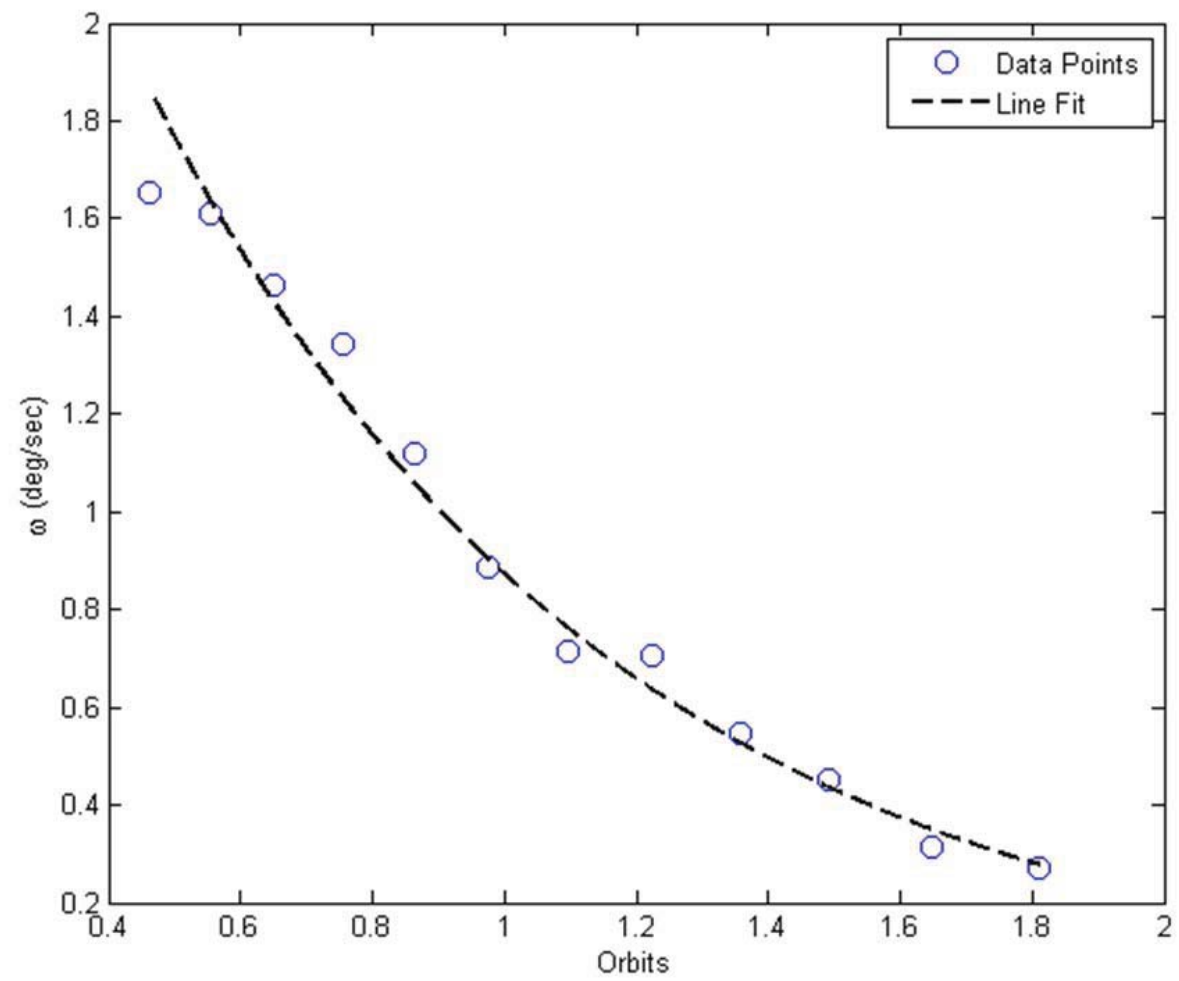

Figure 9. Average magnetic field data point analysis (flight data)

\section{Conclusion}

The average magnetic field of the Earth has been studied and developed with different methods and models. All the methods shown in the literature [1] [2] [8] used the mean square error of the magnetic field to determine the average magnetic field. All these mathematical models are dependent on the model of the Earth Magnetic Field which is very well known. However, the b-dot average magnetic field is a very simple method that uses a common rate controller. It does not require complex mathematical model to determine the average magnetic field, and it only uses five simple steps to calculate the average magnetic field per moment of inertia of the vehicle.

Because this method uses the b-dot controller, the flight data can be downloaded in the first pass, and the rates can be analyzed to update the flight computer with a new average magnetic field. As shown in the paper, the simulated average magnetic field is close related to the value determine with the flight data (Equation (15) and Equation (16)).

The main advantage of this method is that the average magnetic field per moment of inertia is the same in the three axes of the satellite. This presents an advantage in the solution of Euler's equation. If Euler's equation is used for a control scheme as its shown in the literature,

$$
\boldsymbol{I} \dot{\vec{\omega}}+\vec{\omega} \times I \vec{\omega}=\vec{m} \times \vec{b}_{E C I}
$$


the control solution for the gains is always related to the mean square error of the average magnetic field [1] [2]. Using this new method to determine the average magnetic field, the applied dipole moments can be related as the opposite of each other so that Euler's equation can be written as,

$$
\dot{\vec{\omega}}+I^{-1} \vec{\omega} \times I \vec{\omega}=2 \frac{B_{\text {ave }}}{I} \vec{m}
$$

This form of Euler's equation can be used to determine the control gains or to develop a controller that does not require the use of the mean square error as shown in the literature [1] [2]. This form of Euler's equation will be subject of future work.

In conclusion, the process shown in this paper to determine the average magnetic field can be very easily implemented and demonstrated with a b-dot controller. Also, the FASTSAT's flight data validates this new method which provides interesting results for the development of future attitude controllers based on the magnetic field. As shown in the solution, this method based on the b-dot controller reduces the mathematical complexity shown in the literature.

\section{References}

[1] Psiaki, Mark L. , "Magnetic Torquer Attitude Control via Asymptotic Periodic Linear Quadratic Regulation", Journal of Guidance, Control, and Dynamics, Vol. 24, Issue no. 2, pp. 386-394, MarchApril 2001.

[2] Lovera, Marco and Astolfi, Alessandro, "Global Magnetic Attitude Control of Spacecraft in the Presence of Gravity Gradient", IEEE Transactions on Aerospace and Electronic Systems, Vol. 42, Issue no. 3, pp. 796-806, July 2006.

[3] Capo-Lugo, Pedro A and Bainum, Peter M. , Orbital Mechanics and Formation Flying, A Digital Control Perspective, Cambridge, UK, Woodhead Publishing, 2011.

[4] Nair, Manoj. (2010, January) International Geomagnetic Reference Field. [Online]. http://www.ngdc.noaa.gov/IAGA/vmod.igrf.html

[5] Wertz, James R. , Attitude Determination and Control, Dordecht, Holland, Reidel Publishing Company, 2002.

[6] Maus, S. et al., "The 10th Generation International Geomagnetic Reference Field", Physics of the Earth and Planetary Interiors, Vol. 151, pp. 320-322, 2005.

[7] Gravdahl, Jan Tommy, "Magnetic Attitude Control for Satellites", in 43rd IEEE Conference on Decision and Control, Atlantis, Paradise Island, Bahamas, December 14-17,2004.

[8] Wisniewski, Rafa , "Linear Time Varying Approach to Satellite Attitude Control Using Only Electromagnetic Actuation", , 1997,AIAA-97-3479. 
[9] Wertz, James R. and Larson, Wiley J. , Space Mission Analysis and Design, 3rd ed., California, USA, Microcosm Press and Kluwer Academic Publishers, 1999.

[10] Reyhanoglu, M. and Hervas, J.R. , "Three-axis magnetic attitude control algorithms for small satellites", in 5th International Conference on Recent Advances in Space Technologies (RAST), Istanbul, Turkey, 2011, pp. 897-902.

[11] Nise, Norman S., Control System Engineering, Fourth Edition ed., USA, John Wiley \& Sons, 2004.

[12] Ogata, Katsuhiko, System Dynamics, 4th ed., New Jersey, USA, Prentice Hall, 2004.

[13] Larson, Ron and Farber, Betsy , Elementary Statistics, Picturing the World, 5th ed., Boston, USA, Prentice Hall, 2012.

[14] Marter, F. , Pal, P.K. , and Psiaki, M. , "Active Magnetic Control System for Gravity Gradient Stabilized Spacecraft", in Proceedings of the 2nd Annual AIAA/USU Conference on Small Satellites, Utah State University, Logan, Utah, 1988.

[15] Steyn, William H. , "Comparison of Low-Earth-Orbit Satellite Attitude Controller Submitted to Controllability Constraints", Journal of Guidance, Control, and Dynamics, Vol. 17, Issue no. 4, pp. 795-804, July-August 1994.

[16] DeKock, Brandon, Sanders, Devon, VanZwieten, Tannen, and Capo-Lugo, Pedro, "Design and Integration of an All-Magnetic Attitude Control System for FASTSAT-HSV01 Multiple Pointing Objectives", in 34th Annual Guidance, Navigation, and Control Conference, Breckenridge, Colorado, February 4-9, 2011,AAS 11-034. 


\section{Appendix A. Proof of Equation (10) and Equation (12)}

The differential equation obtained from Figure (3a) is,

$$
\dot{\omega}(t)+\xi \omega(t)=\xi \omega_{r}(t)
$$

The time is scaled down by using the following relation,

$$
\tau=t / T
$$

The Scaling theorem of the Laplace transform says that,

$$
\mathcal{L}[f(a t)]=\frac{1}{a} F\left(\frac{s}{a}\right)
$$

$a$ is an arbitrary constant larger than zero. The following relations can be obtained from each term in equation (A.1) using equation (A.3),

$$
\begin{aligned}
& \mathcal{L}\left[\dot{\omega}\left(\frac{t}{T}\right)\right]=T^{2} s \omega(T s) \\
& \mathcal{L}\left[\xi \omega\left(\frac{t}{T}\right)\right]=T \xi \omega(T s) \\
& \mathcal{L}\left[\xi \omega_{r}\left(\frac{t}{T}\right)\right]=T \xi \omega_{r}(T s)
\end{aligned}
$$

Using equations (A.4), the Laplace transform of equation (A.1) is,

$$
\frac{\omega(s T)}{\omega_{r}(s T)}=\frac{\xi / T}{s+\xi / T}
$$

Equation (A.5) is equation (10). Using the Scaling theorem once again, the following Laplace transform can be obtained,

$$
\mathcal{L}\left[e^{-\frac{a t}{T}} u\left(\frac{t}{T}\right)\right]=\frac{1}{s+\frac{a}{T}}
$$

where $u\left(\frac{t}{T}\right)$ is the unit step function. With equation (A.6), the inverse Laplace transform of equation (A.5) equals to,

$$
\omega\left(\frac{t}{T}\right)=\frac{\xi}{T} e^{-\frac{\xi}{T} t} \omega_{r}
$$

Using the relation in equation (A.2), equation (12) is obtained as shown in the following equation.

$$
\omega(\tau)=\frac{\xi}{T} e^{-\xi \tau} \omega_{r}
$$

